\title{
Economic evaluation of therapies for patients suffering from relapsed-refractory multiple myeloma in Greece
}

This article was published in the following Dove Press journal:

Cancer Management and Research

9 April 2013

Number of times this article has been viewed

\section{Fragoulakis' \\ E Kastritis ${ }^{2}$ \\ T Psaltopoulou ${ }^{3}$ \\ N Maniadakis'}

'National School of Public Health, Athens, Greece; ${ }^{2}$ Department of Clinical Therapeutics, Alexandra Hospital, University of Athens, School of Medicine, Athens, Greece; ' ${ }^{3}$ Department of Hygiene, Epidemiology and Medical Statistics, University of Athens, School of Medicine, Athens, Greece
Correspondence: Vassilis Fragoulakis Department of Health Services and Management, National School of Public Health, Alexandras Av 196,

Athens II52I, Greece

Tel +302132010207

$\mathrm{Fax}+302132010194$

Emailvfragoulakis@yahoo.com
Background: Multiple myeloma is a hematologic malignancy that incurs a substantial economic burden in care management. Since most patients with multiple myeloma eventually relapse or become refractory to current therapies (rrMM), the aim of this study was to assess the costeffectiveness of the combination of lenalidomide-dexamethasone, relative to bortezomib alone, in patients suffering from rrMM in Greece.

Methods: An international discrete event simulation model was locally adapted to estimate differences in overall survival and treatment costs associated with the two alternative treatment options. The efficacy data utilized came from three international trials (MM-009, MM-010, APEX). Quality of life data were extracted from the published literature. Data on resource use and prices came from relevant local sources and referred to 2012. The perspective of the analysis was that of public providers. Total costs for monitoring and administration of therapy to patients, management of adverse events, and cost of medication were captured. A 3.5\% discount rate was used for costs and health outcomes. A Monte Carlo simulation was used to estimate probabilistic results with 95\% uncertainty intervals (UI) and a cost-effectiveness acceptability curve.

Results: The mean number of quality-adjusted life years (QALYs) was 3.01 (95\% UI 2.81-3.20) and 2.22 (95\% UI 2.02-2.41) for lenalidomide-dexamethasone and bortezomib, respectively, giving an incremental gain of 0.79 (95\% UI 0.49-1.06) QALYs in favor of lenalidomide-dexamethasone. The mean cost of therapy per patient was estimated at $€ 77,670$ (95\% UI €76,509-€78,900) and €48,928 (95\% UI €48,300-€49,556) for lenalidomidedexamethasone and bortezomib, respectively. The incremental cost per life year gained with lenalidomide-dexamethasone was estimated at $€ 29,415$ (95\% UI $€ 23,484-€ 37,583)$ and the incremental cost per QALY gained at $€ 38,268$ (95\% UI $€ 27,001-€ 58,065$ ). The probability of lenalidomide-dexamethasone being a cost-effective therapy option at a threshold three times the per capita income ( $€ 60,000$ per QALY) was higher than $95 \%$. The results remained constant, without altering the conclusions, under several hypothetical scenarios.

Conclusion: The combination of lenalidomide and dexamethasone may represent a costeffective choice relative to bortezomib monotherapy for patients in Greece with previously treated multiple myeloma.

Keywords: multiple myeloma, economic evaluation, lenalidomide, dexamethasone, bortezomib

\section{Introduction}

Multiple myeloma is a malignancy of the plasma cells that normally produce the antibodies directed against pathogens and other foreign antigens. Multiple myeloma affects both genders and represents the second most common hematologic malignancy. ${ }^{1,2}$ On a worldwide scale, it has been estimated that multiple myeloma accounts for $0.8 \%$ 
of all new cancer cases and $0.9 \%$ of all cancer deaths. ${ }^{3}$ Based on data from the GLOBOCAN database, the incidence of multiple myeloma in Europe is close to 37,000 cases, and the disease accounts for approximately 24,000 deaths per year. ${ }^{2}$ In Greece, the five-year prevalence for the adult population has been estimated at 1240 patients, while the incidence of the disease has been estimated at 500 new patient cases per year. $^{2}$

Multiple myeloma may be considered a disease of the elderly, with a median age at diagnosis of 65-70 years. ${ }^{4}$ Despite the fact that survival tends to be longer in younger patients, accurate health outcome data for patients in different age groups have not yet been reported. ${ }^{5}$

Patients with multiple myeloma often suffer from pronounced symptoms and a substantially reduced quality of life. ${ }^{11}$ In particular, multiple myeloma is associated with bone pain, fatigue, infectious complications, and reduced physical function. ${ }^{12}$ The goal of treatment is to achieve disease remission for the longest possible period while maintaining the best possible quality of life. However, there are still few randomized clinical trials in multiple myeloma which include quality of life as a study endpoint. ${ }^{13}$ This may be a disadvantage for patients, because the systematic incorporation of quality of life measurements into clinical trials could allow comparison of treatments based on patient health preferences and health status, amongst other considerations. $^{11}$

Currently, there is still no cure for multiple myeloma, so after an initial response, most patients eventually relapse or become refractory to current therapies (rrMM) and die from progression of myeloma. Corticosteroids, such as dexamethasone and prednisone, and/or chemotherapeutic agents, such as melphalan and doxorubicin, combined with autologous stem cell transplantation, have been the standard therapy option for several decades.

In the late 1990s, thalidomide showed clinical benefits in patients with rrMM, and this was followed by introduction of other novel agents with a significantly superior effect. ${ }^{10}$

In particular, lenalidomide, an immunomodulating agent, and bortezomib, a proteasome inhibitor, showed a significant effect in rrMM. In several randomized Phase III studies, their use resulted in significant improvements in terms of progression-free survival, overall survival, and quality of life in patients previously treated with thalidomide. Accordingly, both lenalidomide and bortezomib have been approved by the US Food and Drug Administration and European Medicines Agency for the treatment of patients with rrMM and have become standard therapy options. ${ }^{6-9,14}$
Thalidomide, bortezomib, and lenalidomide have significant differences in their mechanisms of action and effect and, more importantly, they have different toxicity profiles. More specifically, bortezomib is a proteasome inhibitor that is given intravenously (the subcutaneous route was approved very recently), and is associated with a significant risk of neurotoxicity (peripheral neuropathy) and reactivation of herpes zoster, while it is only mildly myelosuppressive. Lenalidomide is an immunomodulatory drug given orally once a day, and is associated with an increased risk of myelosuppression and thromboembolism, and also with very low rates of neurotoxicity.

Multiple myeloma accounts for only a small portion of all cancer cases; however, due to the price of the aforementioned medications, the mean total cost of managing patients is quite significant. ${ }^{15}$ Therefore, in general, it is important to assess the cost-effectiveness ratio of therapies in order to ascertain better whether they represent a rational investment of scarce resources. Moreover, at present, Greece is going through a major economic crisis, probably the most significant in its modern history, and in such a context it is paramount to ensure that health care resources are spent wisely on therapies that maximize the health benefit. In this context, an economic evaluation was undertaken to assess the cost-effectiveness of lenalidomide in combination with dexamethasone versus bortezomib alone for the treatment of patients with rrMM in Greece. This paper presents the results of this economic analysis.

\section{Materials and methods Aim and perspective of analysis}

The aim of this study was to compare the standard therapies used in patients with rrMM locally. These are also expensive therapies, so their choice must be justified on economic grounds. Other comparisons were not undertaken because they do not correspond to commonly used approaches. Moreover, given that the analysis is based on specific clinical trials, treatments given in the initial setting are assumed, and the present results and conclusions should be assessed in that light. In addition, based on expert opinion, the population of the trials was assumed to be similar to that of the local population.

The perspective of analysis is the point of view from which the costs and benefits are recorded and evaluated. It is decided based on the research question and the decision it is designed to support. It can be that of the health care system, of payers, or society overall. The choice defines the way outcomes are quantified. In the present evaluation, 
the perspective of the analysis was that of the public health care system in Greece. With regard to the salaries of medical staff, providers are funded directly from the state budget, so this cost item was not considered, because the marginal cost of caring for this group of patients is zero. The analysis considered the operational expenses associated with the direct health care management of patients with rrMM.

In general, the resources considered concern the drugs, consumables, devices, and diagnostic, laboratory, and radiology tests, as well as inpatient stay, that are utilized in the delivery of each therapy under consideration and to manage associated adverse events. Costs associated with treating comorbidities are not considered, because they were assumed to be equal across therapy arms, and according to economic evaluation guidelines can be excluded, because they do not have an impact on the final incremental result. Other costs that represent the indirect burden, such as the productivity loss associated with each therapy, or direct payments by patients for traveling or other resources, are harder to quantify accurately and thus were not considered in the present analysis.

\section{Model and data}

The model used in this assessment represents a local adaptation of the pharmacoeconomic model submitted to the National Institute of Health and Clinical Excellence in the UK by the manufacturer of lenalidomide for assessment. This model has a similar structure and has incorporated estimation of mean survival of comparators, adjusted with the local discount rate, from a recently published costeffectiveness model and analysis undertaken for the case of Norway. ${ }^{16}$ A discrete event simulation model was adopted, in the context of which every simulated individual is assigned the specific characteristics of actual patients based on trial records. Because they are based on individual data patterns, the advantage of a discrete event simulation model is that it provides greater flexibility compared with simple Markov models, in an effort to assess patients with rrMM in terms of time to response, duration of response, and survival. ${ }^{17}$

The model simulates the progress and outcomes of a large hypothetical patient population (eg, 1000), consisting of individuals with characteristics and clinical histories similar to those of patients enrolled in the MM-009 and MM-010 trials. ${ }^{18,19}$ MM-009 and MM-010 were two identical multicenter, pivotal, double-blind, placebo-controlled, randomized, Phase III trials comparing patients receiving $25 \mathrm{mg}$ of daily oral lenalidomide or placebo, plus $40 \mathrm{mg}$ oral dexamethasone in both arms. Pooled data analysis of these trials (346 patients in each arm) revealed that treatment with lenalidomide-dexamethasone significantly improved the overall response rate $(60.6 \%$ versus $21.9 \%)$, complete response rate $(15.0 \%$ versus $2.0 \%)$, time to progression (13.4 versus 4.6 months), and duration of response (15.8 versus 7 months) compared with dexamethasone monotherapy. ${ }^{20}$ At a median follow-up of 48 months for surviving patients, a significant benefit in overall survival (median of 38.0 versus 31.6 months) was also observed. This led to the approval of the combination in patients with raM in several countries worldwide.

In the case of bortezomib, efficacy data were extracted from the open-label APEX trial. ${ }^{21}$ This study compared bortezomib with high-dose dexamethasone in patients with rrMM who had received one to three previous therapies (333 versus 336 patients). Median survival was estimated at 29.8 months for the bortezomib arm versus 23.7 months for dexamethasone. Overall response rates were $43 \%$ for the bortezomib arm and $9 \%$ for the dexamethasone treatment arm..$^{22}$ Given that there were no significant differences between patient characteristics in the MM-009/-010 and APEX trials, an indirect comparison between lenalidomide-dexamethasone versus bortezomib was considered to be a feasible and reasonable approach for the purposes of an economic evaluation, in the absence of direct comparison studies.

The response of patients was assessed according to the criteria of the European Group for Blood and Marrow Transplant. ${ }^{23}$ The model considered four response levels, in accordance with clinical trials, ie, complete response, partial response, stable disease, and progressive disease. The model is based on the trial results and simulates patients for their entire lifetime, hence it extrapolates beyond the trial horizon, which is a common approach in modeling studies. Specifically, in the discrete event model, a predefined number of patients with multiple myeloma, each with distinctive characteristics (eg, age, gender, number of prior treatments), are selected randomly from patients who were enrolled in the clinical trials. The patients from the trials are pooled, regardless of trial or treatment assignment, to create one large population. This population is then subdivided into four datasets, one for each best-response category, ie, complete response, partial response, stable disease, and progressive disease, again irrespective of treatment, assuming that a given response achieved by using different treatments is clinically the same and the impact on the patient's disease course will be the same. Thus, the model does not favor any response level achieved by one treatment over the same response level achieved by another treatment, but focuses 
on the difference in the proportions of patients achieving a response. The model randomly selects patients from the appropriate patient file based on the response distribution for each treatment. For example, if a total of 1000 patients are to be simulated and $10 \%$ have a complete response to treatment with lenalidomide-dexamethasone, then the model randomly picks 100 patients from the complete response file and assigns them to treatment with lenalidomide-dexamethasone. For each patient, the model compares the times of all the possible events and processes the events chronologically, for each individual patient it is allowed to follow a specific course of disease that is reflective of that patient's characteristics and treatment options. The model selects the event with the earliest occurrence time and initiates processing of that event at the appropriate time. Any times that exceed the model's end time indicate that the particular event will not occur during that simulation timeframe. Similarly, event times that exceed life expectancy indicate that death will precede, and therefore preclude, those events. In particular, the "events" that are considered in the model, apart from the "adverse events" which are mentioned later, are the following: "progression", "next observation", “death", "model end", and "end of treatment". Before processing the next event, the simulation accrues the quality-adjusted life years (QALYs) lived according to the patient's current utility and the time to the next event. The model also keeps track of the accumulating monitoring costs depending on being in a relapsed or nonrelapsed state.

The main outcomes considered in the model were median time to progression, overall survival, mean therapy cost, cost per QALY, and cost per life year. A 3.5\% rate was used to discount therapy costs, QALYs, and life years, in line with similar studies undertaken for Greece. ${ }^{24,25}$ The model projected "infinitely" in the future (ie, up to 100 years of age for any simulated individual) to capture all outcomes during the patients' remaining lifetime.

\section{Risk equations}

The overall survival of patients was determined by time to progression and post-progression survival. The structure of the modeled overall survival in both arms is depicted in Figure 1. Time to progression and post-progression survival, both affected by response levels and individual patient characteristics, were analyzed via a parametric failure-time analysis. The time to progression equation was derived in two steps. In the first step, the best fitting distribution was identified. Amongst several distributions, the Weibull distribution was identified as the best fitting

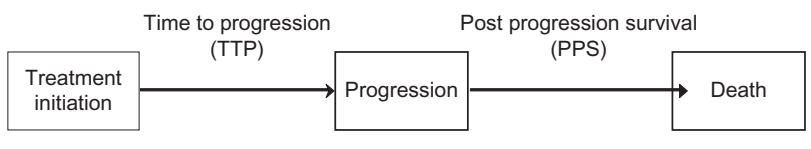

\section{Overall survival $=$ TTP + PPS}

Figure I Structure of model.

option based on a minus-2-log-likelihood criterion. This type of distribution allowed for estimation of the probability of an event occurring during different time intervals after the imitation point. The hazard function used consists of two parameters, a scale parameter called lambda $(\lambda)$, and a shape parameter called sigma $(\sigma)$, that determine how the hazard develops over time. Progression-free time was measured from the date of randomization to the date of confirmed progression. If no event occurred, the subject's time was considered right-censored and the study end date or last observation was taken as the time of censoring. The second step for constructing the risk equation for time to progression consisted of selecting significant predictors. These were included as baseline characteristics, as well as the best response achieved by patients prior to progression. Variables that had no significant association with time to progression were removed iteratively, until a final model that included only significant predictors was selected.

A similar approach was used to fit post-progression survival. For the case of post-progression survival, a Weibull distribution with shape parameter equal to 1 was used. To estimate the individual post-progression survival, a hazard was calculated with the characteristics of each patient and then used to estimate the time of death, if not observed in the trial, by sampling from the resulting failure time distribution. Table 1 presents in detail the coefficients for predicted time (in months) to progression and post-progression survival for patients with rrMM.

\section{Adverse events}

The following adverse events were considered in the model: anemia, thrombocytopenia, neutropenia, hypocalcemia, diarrhea, constipation, pneumonia, peripheral neuropathy, and deep vein thrombosis. Based on expert advice and the literature, it was assumed that only grades III and IV adverse events had a significant resource and cost impact, so grades I and II were not included in this assessment. ${ }^{16}$ The rate of adverse events was expressed as events per person-month for each three-month (90-day) interval. To facilitate simulation of occurrence of the events, these rates were converted to 
Table I Coefficients of post-progression survival and time to progression in the Weibull model

\begin{tabular}{|c|c|c|c|c|}
\hline \multirow[t]{2}{*}{ Parameter } & \multicolumn{2}{|c|}{$\begin{array}{l}\text { Time to } \\
\text { progression }\end{array}$} & \multicolumn{2}{|c|}{$\begin{array}{l}\text { Post-progression } \\
\text { survival }\end{array}$} \\
\hline & Len-Dex & Bort & Len-Dex & Bort \\
\hline Shape & 1.76 & 1.76 & 1.00 & 1.00 \\
\hline Intercept & 1.78 & 1.78 & 4.15 & 4.60 \\
\hline Treated & 0.38 & - & & \\
\hline$C R$ versus $S D$ & 2.39 & 2.39 & 0.49 & 0.49 \\
\hline PR versus SD & 1.01 & 1.01 & 0.49 & 0.49 \\
\hline PD versus SD & -0.85 & -0.85 & -0.39 & -0.39 \\
\hline Treated * PD & 1.25 & - & - & - \\
\hline Beta-2M > 2.5 & -0.46 & -0.46 & -1.14 & -1.14 \\
\hline Prior stem cell transplant & -0.31 & -0.31 & - & - \\
\hline Disease duration (years) & 0.11 & 0.11 & 0.06 & 0.06 \\
\hline Lytic bone lesions $(\mathrm{Y} / \mathrm{N})$ & - & - & -0.67 & -0.67 \\
\hline ECOG $2-3$ versus $0-1$ & - & - & -0.64 & -0.64 \\
\hline $\begin{array}{l}\text { Multiple versus one prior } \\
\text { therapy }\end{array}$ & - & - & -0.24 & -0.24 \\
\hline
\end{tabular}

three-month probabilities. Table 2 presents in detail the type of adverse event per person-month for each interval.

\section{Cost assessment methodology}

In the lenalidomide-dexamethasone arm, it was assumed that all patients received a $25 \mathrm{mg}$ dose of lenalidomide for 21 days of each 28-day cycle until disease progression was observed. The dose of dexamethasone was $40 \mathrm{mg}$ /day on days $1-4$, days $9-12$, and days 17-20 for four cycles, and after four cycles, the dose of dexamethasone was $40 \mathrm{mg} /$ day on days $1-4$. In the case of bortezomib, a 21-day cycle for eight cycles was assumed. Bortezomib dosing was $1.3 \mathrm{mg} / \mathrm{m}^{2}$ per dose, administered as a bolus intravenous injection twice weekly for 2 weeks (days 1, 4, 8, and 11) followed by a 10-day rest period. The cost of lenalidomide was $€ 5186.77$ for a $25 \mathrm{mg}$ capsule and $€ 864.98$ per vial of bortezomib, while the cost of dexamethasone was $€ 0.27$ per mg. The hospital prices of drugs used in the model were obtained from the last price bulletin ${ }^{26}$ issued by the Ministry of Health (March 2012) and are applicable across all hospitals in Greece.

The cost of adverse events was calculated by multiplying the probability of occurrence of any event by its management cost. The later incorporated the cost (Table 3 ) of all resources consumed to handle adverse events in the inpatient or outpatient care setting, according to local management practices, outlined by medical experts who reviewed them based on a structured questionnaire. The total treatment cost included drugs and their administration, patient monitoring, and hospitalizations for handing adverse events. The cost of adverse events was applied to those patients experiencing any event in their corresponding treatment arm.

The monitoring costs reflected the fees of laboratory examinations multiplied by their mean frequency according to expert advice. The unit costs of examinations were obtained from the Government Gazette and express the official resource prices in 2012. The study did not evaluate the cost of physicians and other personnel time, because there is no marginal difference, given that they are civil servants who are funded through fixed salaries coming from state budgets. ${ }^{27}$ For patients with rrMM who needed inpatient care, the cost of biochemistry examinations or low-cost drugs (such as aspirin or trimethoprim-sulfamethoxazole) were estimated via the reimbursed cost per hospital day, to avoid double counting. Table 4 summarizes in detail the examination costs used in the model.

\section{Utility scores}

There is very limited information in the literature regarding quality of life in patients with rrMM. In this model, the utility score for nonresponders (with progressive disease), was estimated at 0.64 , based on a study conducted by the Dutch-Belgian Hematology-Oncology Cooperative Study Group. ${ }^{28}$ All other response levels were set at a utility level of 0.81 , based on the same study. Because of lack of data, no other disutility was included for the adverse events.

\section{Uncertainty}

It is generally known that economic data are truncated at zero and do not follow normal distributions; consequently, hypothesis testing would be invalid if conducted with conventional approaches (eg, 95\% confidence intervals). Therefore, in the present model, bias-corrected uncertainty intervals (UI) were calculated using the percentile method of nonparametric simulation. ${ }^{29}$ Probability distributions were specified around the main model parameters. In particular, all cost components were associated with a gamma distribution and the utility values with a beta distribution, and a $10 \%$ of coefficient of variation around the mean was used for probabilistic sensitivity analysis (1000 bootstrap replications). Because the variance-covariance matrix was known, time to progression and overall survival were associated with a normal distribution, while a linear correlation pattern amongst patient characteristics was taken into account in each bootstrap experiment. To address uncertainty related to mean survival per patient group, a $10 \%$ coefficient of variation was assumed for probabilistic sensitivity analysis. In addition, a supplementary one-way sensitivity analysis 
Table 2 Grade 3 and 4 adverse events and complications

\begin{tabular}{|c|c|c|c|c|c|c|c|c|}
\hline & $\begin{array}{l}0-3 \\
\text { months }\end{array}$ & $\begin{array}{l}3-6 \\
\text { months }\end{array}$ & $\begin{array}{l}\text { 6-9 } \\
\text { months }\end{array}$ & $\begin{array}{l}9-12 \\
\text { months }\end{array}$ & $\begin{array}{l}\text { I2-I5 } \\
\text { months }\end{array}$ & $\begin{array}{l}\text { I5-18 } \\
\text { months }\end{array}$ & $\begin{array}{l}|8-2| \\
\text { months }\end{array}$ & $\begin{array}{l}21-24 \\
\text { months }\end{array}$ \\
\hline \multicolumn{9}{|l|}{ Len-Dex } \\
\hline \multicolumn{9}{|l|}{ Grade 3} \\
\hline Anemia & $9.2 \%$ & $12.5 \%$ & $12.5 \%$ & $13.2 \%$ & $13.2 \%$ & $14.0 \%$ & $15.6 \%$ & $15.6 \%$ \\
\hline Thrombocytopenia & $\mathrm{I} 1.2 \%$ & $20.1 \%$ & $20.7 \%$ & $22.1 \%$ & $22.5 \%$ & $22.5 \%$ & $22.5 \%$ & $22.5 \%$ \\
\hline Neutropenia & $22.3 \%$ & $27.8 \%$ & $35.1 \%$ & $39.0 \%$ & $43.4 \%$ & $45.0 \%$ & $46.7 \%$ & $51.4 \%$ \\
\hline Hypercalcemia & $0.0 \%$ & $0.0 \%$ & $0.0 \%$ & $0.0 \%$ & $0.0 \%$ & $0.0 \%$ & $0.0 \%$ & $0.0 \%$ \\
\hline Diarrhea & $2.4 \%$ & $2.4 \%$ & $3.1 \%$ & $3.1 \%$ & $3.1 \%$ & $3.1 \%$ & $3.1 \%$ & $3.1 \%$ \\
\hline Constipation & $2.4 \%$ & $2.4 \%$ & $2.4 \%$ & $2.4 \%$ & $2.4 \%$ & $2.4 \%$ & $2.4 \%$ & $2.4 \%$ \\
\hline Pneumonia & $7.6 \%$ & $17.7 \%$ & $18.6 \%$ & $19.9 \%$ & $20.8 \%$ & $20.8 \%$ & $20.8 \%$ & $20.8 \%$ \\
\hline Peripheral neuropathy & $2.9 \%$ & $4.1 \%$ & $4.8 \%$ & $4.8 \%$ & $4.8 \%$ & $4.8 \%$ & $4.8 \%$ & $4.8 \%$ \\
\hline Deep vein thrombosis & $8.0 \%$ & $13.4 \%$ & $13.8 \%$ & $14.9 \%$ & $14.9 \%$ & $15.6 \%$ & $15.6 \%$ & $15.6 \%$ \\
\hline \multicolumn{9}{|l|}{ Grade 4} \\
\hline Anemia & $0.9 \%$ & $1.3 \%$ & $1.3 \%$ & $1.3 \%$ & $1.3 \%$ & $1.3 \%$ & $1.3 \%$ & $1.3 \%$ \\
\hline Thrombocytopenia & $0.3 \%$ & $1.0 \%$ & $1.0 \%$ & $1.0 \%$ & $1.0 \%$ & $1.0 \%$ & $2.6 \%$ & $2.6 \%$ \\
\hline Neutropenia & $2.5 \%$ & $4.2 \%$ & $4.6 \%$ & $4.6 \%$ & $5.0 \%$ & $5.0 \%$ & $5.0 \%$ & $5.0 \%$ \\
\hline Hypercalcemia & $0.0 \%$ & $0.0 \%$ & $0.0 \%$ & $0.0 \%$ & $0.0 \%$ & $0.0 \%$ & $0.0 \%$ & $0.0 \%$ \\
\hline Diarrhea & $0.0 \%$ & $0.0 \%$ & $0.0 \%$ & $0.0 \%$ & $0.0 \%$ & $0.0 \%$ & $0.0 \%$ & $0.0 \%$ \\
\hline Constipation & $0.0 \%$ & $0.0 \%$ & $0.0 \%$ & $0.0 \%$ & $0.0 \%$ & $0.0 \%$ & $0.0 \%$ & $0.0 \%$ \\
\hline Pneumonia & $0.9 \%$ & $0.9 \%$ & $0.9 \%$ & $0.9 \%$ & $0.9 \%$ & $0.9 \%$ & $0.9 \%$ & $0.9 \%$ \\
\hline Peripheral neuropathy & $0.0 \%$ & $0.0 \%$ & $0.0 \%$ & $0.0 \%$ & $0.0 \%$ & $0.0 \%$ & $0.0 \%$ & $0.0 \%$ \\
\hline Deep vein thrombosis & $0.3 \%$ & $0.3 \%$ & $0.3 \%$ & $0.3 \%$ & $0.3 \%$ & $0.3 \%$ & $0.3 \%$ & $0.3 \%$ \\
\hline \multicolumn{9}{|l|}{ Bortezomib } \\
\hline \multicolumn{9}{|l|}{ Grade 3} \\
\hline Anemia & $2.8 \%$ & $5.6 \%$ & $8.2 \%$ & $9.1 \%$ & $9.1 \%$ & $9.1 \%$ & $9.1 \%$ & $9.1 \%$ \\
\hline Thrombocytopenia & $9.0 \%$ & $17.2 \%$ & $24.7 \%$ & $27.0 \%$ & $27.0 \%$ & $27.0 \%$ & $27.0 \%$ & $27.0 \%$ \\
\hline Neutropenia & $3.8 \%$ & $7.5 \%$ & $11.0 \%$ & $12.2 \%$ & $12.2 \%$ & $12.2 \%$ & $12.2 \%$ & $12.2 \%$ \\
\hline Hypercalcemia & $0.0 \%$ & $0.0 \%$ & $0.0 \%$ & $0.0 \%$ & $0.0 \%$ & $0.0 \%$ & $0.0 \%$ & $0.0 \%$ \\
\hline Diarrhea & $2.2 \%$ & $4.3 \%$ & $6.4 \%$ & $7.1 \%$ & $7.1 \%$ & $7.1 \%$ & $7.1 \%$ & $7.1 \%$ \\
\hline Constipation & $0.6 \%$ & $1.2 \%$ & $1.8 \%$ & $2.0 \%$ & $2.0 \%$ & $2.0 \%$ & $2.0 \%$ & $2.0 \%$ \\
\hline Pneumonia & $0.0 \%$ & $0.0 \%$ & $0.0 \%$ & $0.0 \%$ & $0.0 \%$ & $0.0 \%$ & $0.0 \%$ & $0.0 \%$ \\
\hline Peripheral neuropathy & $2.2 \%$ & $4.3 \%$ & $6.4 \%$ & $7.1 \%$ & $7.1 \%$ & $7.1 \%$ & $7.1 \%$ & $7.1 \%$ \\
\hline Deep vein thrombosis & $0.0 \%$ & $0.0 \%$ & $0.0 \%$ & $0.0 \%$ & $0.0 \%$ & $0.0 \%$ & $0.0 \%$ & $0.0 \%$ \\
\hline \multicolumn{9}{|l|}{ Grade 4} \\
\hline Anemia & $0.3 \%$ & $0.6 \%$ & $0.9 \%$ & $1.0 \%$ & $1.0 \%$ & $1.0 \%$ & $1.0 \%$ & $1.0 \%$ \\
\hline Thrombocytopenia & $1.2 \%$ & $2.4 \%$ & $3.6 \%$ & $3.9 \%$ & $3.9 \%$ & $3.9 \%$ & $3.9 \%$ & $3.9 \%$ \\
\hline Neutropenia & $0.6 \%$ & $1.2 \%$ & $1.8 \%$ & $2.0 \%$ & $2.0 \%$ & $2.0 \%$ & $2.0 \%$ & $2.0 \%$ \\
\hline Hypercalcemia & $0.0 \%$ & $0.0 \%$ & $0.0 \%$ & $0.0 \%$ & $0.0 \%$ & $0.0 \%$ & $0.0 \%$ & $0.0 \%$ \\
\hline Diarrhea & $0.0 \%$ & $0.0 \%$ & $0.0 \%$ & $0.0 \%$ & $0.0 \%$ & $0.0 \%$ & $0.0 \%$ & $0.0 \%$ \\
\hline Constipation & $0.0 \%$ & $0.0 \%$ & $0.0 \%$ & $0.0 \%$ & $0.0 \%$ & $0.0 \%$ & $0.0 \%$ & $0.0 \%$ \\
\hline Pneumonia & $0.0 \%$ & $0.0 \%$ & $0.0 \%$ & $0.0 \%$ & $0.0 \%$ & $0.0 \%$ & $0.0 \%$ & $0.0 \%$ \\
\hline Peripheral neuropathy & $0.0 \%$ & $0.6 \%$ & $0.9 \%$ & $1.0 \%$ & $1.0 \%$ & $1.0 \%$ & $1.0 \%$ & $1.0 \%$ \\
\hline Deep vein thrombosis & $0.0 \%$ & $0.0 \%$ & $0.0 \%$ & $0.0 \%$ & $0.0 \%$ & $0.0 \%$ & $0.0 \%$ & $0.0 \%$ \\
\hline
\end{tabular}

Note: Based on MM09-10 and APEX trial.

was conducted. Hence, all the main parameters of the model were varied at $\pm 10 \%$ to examine the stability and robustness of results under different assumptions.

\section{Results}

Table 5 presents the model outcomes (mainly cost, life years, QALYs, time to progression) by therapy arm. All outcomes are reported at discounted values. According to the analysis, lenalidomide-dexamethasone provided better clinical outcomes compared with bortezomib in patients who had received at least one prior therapy.
The model predicted median time to progression for bortezomib at 6.73 (95\% UI 6.09-7.08) compared with 14.08 (95\% UI 13.83-14.32) for the lenalidomide-dexamethasone group. The difference in time to progression was estimated at 7.35 (95\% UI 6.84-7.92) months in favor of lenalidomidedexamethasone. The estimated mean number of QALYs in the case of lenalidomide-dexamethasone was 3.01 (95\% UI 2.81-3.20) and 2.22 (95\% UI 2.02-2.41) in the case of bortezomib. Consequently, there was a statistically significant difference of 0.79 (95\% UI 0.49-1.06) QALYs in favor of lenalidomide-dexamethasone. Similarly, the estimated 
Table 3 Cost of adverse events by location of care

\begin{tabular}{|c|c|c|c|c|}
\hline & \multicolumn{2}{|c|}{$\begin{array}{l}\text { Management cost } \\
(€)\end{array}$} & \multicolumn{2}{|c|}{$\begin{array}{l}\text { Frequency of care } \\
\text { by location (\%) }\end{array}$} \\
\hline & Inpatient & Outpatient & Inpatient & Outpatient \\
\hline \multicolumn{5}{|l|}{ Disease-related complications } \\
\hline Anemia grade 3 & 1885 & 1800 & 8.3 & 91.7 \\
\hline Anemia grade 4 & 2085 & 1800 & 11.0 & 89.0 \\
\hline Hypercalcemia grade 3 & 285 & 200 & 20.0 & 80.0 \\
\hline Hypercalcemia grade 4 & 285 & 200 & 20.0 & 80.0 \\
\hline Pneumonia grade 3 & 667 & 10 & 90.0 & 10.0 \\
\hline Pneumonia grade 4 & 934 & - & 100.0 & - \\
\hline \multicolumn{5}{|l|}{ Adverse events } \\
\hline Thrombocytopenia grade 3 & 514 & - & 6.7 & 93.3 \\
\hline Thrombocytopenia grade 4 & 1253 & 1253 & 30 & 70.0 \\
\hline Neutropenia grade 3 & 381 & 160 & 73 & 27.0 \\
\hline Neutropenia grade 4 & 634 & - & 100.0 & - \\
\hline Diarrhea grade 3 & 154 & 3 & 50.0 & 50.0 \\
\hline Diarrhea grade 4 & 235 & - & 100.0 & - \\
\hline Constipation grade 3 & 91 & 6 & 5.0 & 95.0 \\
\hline Constipation grade 4 & 226 & - & 100.0 & - \\
\hline Peripheral neuropathy grade 3 & - & 10 & - & 100.0 \\
\hline Peripheral neuropathy grade 4 & - & 10 & - & 100.0 \\
\hline Deep vein thrombosis grade 3 & 331 & 111 & 30.0 & 70.0 \\
\hline Deep vein thrombosis grade 4 & 478 & 111 & 30.0 & 70.0 \\
\hline
\end{tabular}

mean life years was 4.14 (95\% UI 4.06-4.21) and 3.15 (95\% UI 2.93-3.38) for lenalidomide-dexamethasone and bortezomib, respectively. The difference in life years between comparators was estimated at 0.99 (95\% UI 0.76-1.22) in favor of lenalidomide-dexamethasone.

The total cost was estimated at $€ 77,670$ (95\% UI $€ 76,509-€ 78,900)$ for lenalidomide-dexamethasone and $€ 48,928$ (95\% UI €48,300-€49,556) for bortezomib, an incremental difference of $€ 28,741$ (95\% UI $€ 27,755-€ 29,787)$. The main component driving the total cost of treatment in both arms was the cost of medication. Particularly in the case of lenalidomide-dexamethasone, the cost of medication was the main cost component and accounted for $89.4 \%$ of the total cost. The mean cost of medication in patients receiving lenalidomide-dexamethasone was $€ 69,450$ (95\% UI $€ 68,308-€ 70,550)$, while in those receiving bortezomib it was $€ 40,902$ (95\% UI $€ 40,374-€ 41,448)$, an incremental difference of $€ 28548$ (95\% UI €27,623-29,522). Monitoring costs were estimated at €2943 (95\% UI €2795-€3107) for lenalidomide-dexamethasone and €3324 (95\% UI €3141$€ 3518$ ) for bortezomib, ie, a decrement of $€ 381$ (95\% UI $€ 267-€ 494)$ in favor of lenalidomide-dexamethasone. The cost of adverse events was lower in the case of bortezomib, namely €4702 (95\% UI €4472-€4934), against €5276 (95\% UI €5082-€5485) for lenalidomide-dexamethasone. The incremental cost per life year gained was estimated at $€ 29,415(95 \%$ UI $€ 23,484-€ 37,583)$ and the incremental cost per QALY gained at €38,268 (95\% UI €27,001-€58,065) for lenalidomide-dexamethasone versus bortezomib.

The results from the probabilistic sensitivity analysis are plotted as pairs of differences in costs and effects in the cost-effectiveness plane (Figure 2). The figure shows the results of 1000 simulations, for which the parameter values were changed each time at random based on the assigned distributions. Each dot represents a certain simulation result concerning the difference in costs and the difference in effects. The depicted ellipse was calculated based on the assumption that cost and effects followed the bivariate normal distribution and its contour represented the 95\% uncertainty intervals. All the simulation experiments fell into the southwest quadrant, indicating that the lenalidomidedexamethasone combination was more expensive but also more effective than bortezomib.

Nonetheless, the cost-effectiveness of lenalidomidedexamethasone is a subjective assessment and depends on the willingness to pay a given amount per QALY gained. A convenient way of illustrating the results is the costeffectiveness acceptability curve (Figure 3 ), which shows the chances that a treatment is cost-effective relative to another for different willingness-to-pay levels. The cost-effectiveness acceptability curve shows the probability (on the y-axis) that lenalidomide-dexamethasone may be cost-effective compared with bortezomib for a range (on the x-axis) of maximum monetary values that a decision-maker might be willing 
Table 4 Cost $(€)$ for laboratory examinations and monitoring

\begin{tabular}{|c|c|}
\hline & $\begin{array}{l}\text { Cost } \\
\text { (per test) }\end{array}$ \\
\hline \multicolumn{2}{|l|}{ Laboratory tests/monitoring } \\
\hline Routine blood counts & 2.88 \\
\hline \multicolumn{2}{|l|}{ Clotting } \\
\hline INR & 4.50 \\
\hline \multicolumn{2}{|l|}{ Biochemistry } \\
\hline Liver function tests & 3.00 \\
\hline Erythrocyte sedimentation rate & 3.00 \\
\hline Plasma viscosity & 3.00 \\
\hline Uric acid (urate) & 3.00 \\
\hline Immunoglobulin & 11.80 \\
\hline Paraprotein measurements & 11.80 \\
\hline Protein electrophoresis & 11.80 \\
\hline Serum $\beta 2$ microglobulin & 11.80 \\
\hline C-reactive protein & 13.50 \\
\hline Serum erythropoietin level & 7.20 \\
\hline Serum immunofixation & 13.20 \\
\hline Creatinine clearance & 3.00 \\
\hline Glomerular filtration rate & 3.00 \\
\hline \multicolumn{2}{|l|}{ Routine urinalysis } \\
\hline 24-hour urine measurement & 1.80 \\
\hline 24-hour urine for creatinine & 1.80 \\
\hline 24-hour total urine protein & 6.00 \\
\hline Urine protein electrophoresis/light chains & 6.00 \\
\hline Urine immunofixation & 6.00 \\
\hline \multicolumn{2}{|l|}{ Diagnostics } \\
\hline Skeletal survey by $\mathrm{x}$-ray & 4.00 \\
\hline Skeletal survey by $\mathrm{x}$-ray individual sites & 60.00 \\
\hline Magnetic resonance imaging & 116.16 \\
\hline Bone marrow aspirate & 50.0 \\
\hline Bone marrow trephine biopsy & 71.00 \\
\hline \multicolumn{2}{|l|}{ Other tests } \\
\hline Bacterial investigation & 5.00 \\
\hline Lactic dehydrogenase & 3.00 \\
\hline Calcium & 3.00 \\
\hline Magnesium & 3.00 \\
\hline Hospitalization per day & 85.00 \\
\hline
\end{tabular}

to pay per QALY. According to Figure 2, the probability of lenalidomide-dexamethasone being cost-effective increases significantly at a willingness-to-pay threshold in the range of $€ 40,000$ to $€ 50,000$ per QALY, used in many jurisdictions; notably, at $€ 60,000$ per QALY its probability of cost-effectiveness is higher than 95\%. Table 6 represents the results of the one-way sensitivity analysis. In general, the most sensitive parameter concerning the incremental cost per QALY was the cost of lenalidomide-dexamethasone. Other parameters, such as the cost of other components or the utility values, had less impact on determination of cost per QALY.

\section{Discussion}

Understanding the relative benefits and costs of alternative treatment strategies for patients with rrMM is crucial in order to ensure that patients receive effective and efficient therapy
Table 5 Results of analysis*

\begin{tabular}{lll}
\hline Mean $(95 \%$ UI) & Len-Dex & Bort \\
\hline Health outcomes & & \\
Median time to & 14.08 & 6.73 \\
progression, months & $(|3.83-| 4.32)$ & $(6.09-7.08)$ \\
Mean quality-adjusted & $3.0 I$ & 2.22 \\
life years & $(2.8 I-3.20)$ & $(2.02-2.4 I)$ \\
Mean life years & 4.14 & 3.14 \\
& $(4.06-4.2 I)$ & $(2.93-3.37)$ \\
Economic outcomes & & \\
Total therapy cost & $€ 77,670$ & $€ 48,928$ \\
& $(€ 76,509-€ 78,900)$ & $(€ 48,300-€ 49,556)$ \\
Medication & $€ 69,450$ & $€ 40,902$ \\
& $(€ 68,308-€ 70,550)$ & $(€ 40,374-€ 4 I, 448)$ \\
Monitoring & $€ 2943$ & $€ 3324$ \\
& $(€ 2795-€ 3 \mid 07)$ & $(€ 3|4|-€ 35 \mid 8)$ \\
Adverse event & $€ 5276$ & $€ 4702$ \\
management & $(€ 5082-€ 5485)$ & $(€ 4472-€ 4934)$ \\
Cost-effectiveness analysis & & \\
Cost per QALY & $€ 38,268$ & \\
gained & $(€ 27,00 I-€ 58,065)$ & \\
Cost per life year & $€ 29,4 I 5$ & \\
gained & $(€ 23,484-€ 37,583)$ & \\
\hline
\end{tabular}

Note: *Discounting outcomes at a $3.5 \%$ discount rate.

Abbreviations: QALY, quality-adjusted life years; UI, uncertainty interval.

from the limited health care resources available. This analysis showed that lenalidomide-dexamethasone may be associated with a higher cost, but may also be associated with greater effectiveness compared with its main comparator for patients with rrMM. Until now, there has been no clear official threshold of willingness to pay for a QALY, and important organizations, such as the National Institute of Health and Clinical Excellence, are still seeking the "optimal" threshold for the UK. ${ }^{30}$ Nonetheless, sums between $£ 20,000$ and $£ 30,000$ per QALY are quite often used ${ }^{31}$ and the amount of three times

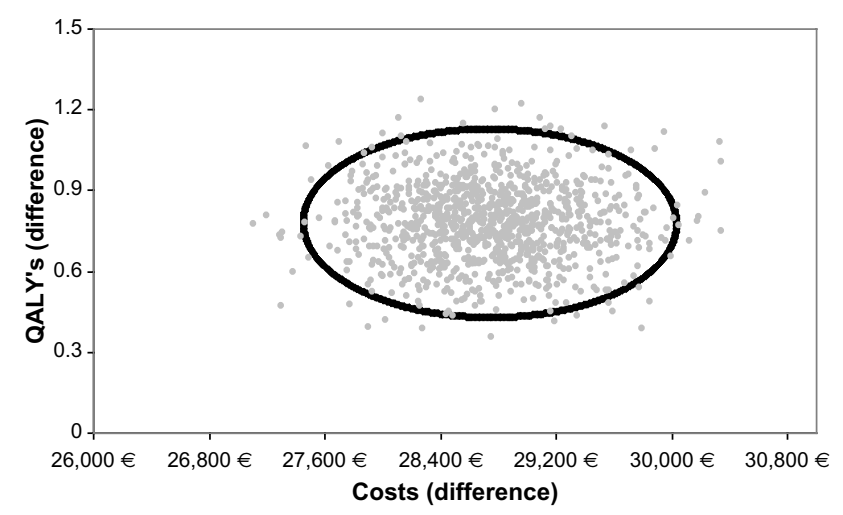

Figure 2 Scatter plot of incremental effect (quality-adjusted life years) and costs between lenalidomide-dexamethasone versus bortezomib.

Notes: Ellipse represents the $95 \%$ bivariate normal distribution; each dot represents a bootstrap experiment.

Abbreviation: QALY, quality-adjusted life years. 


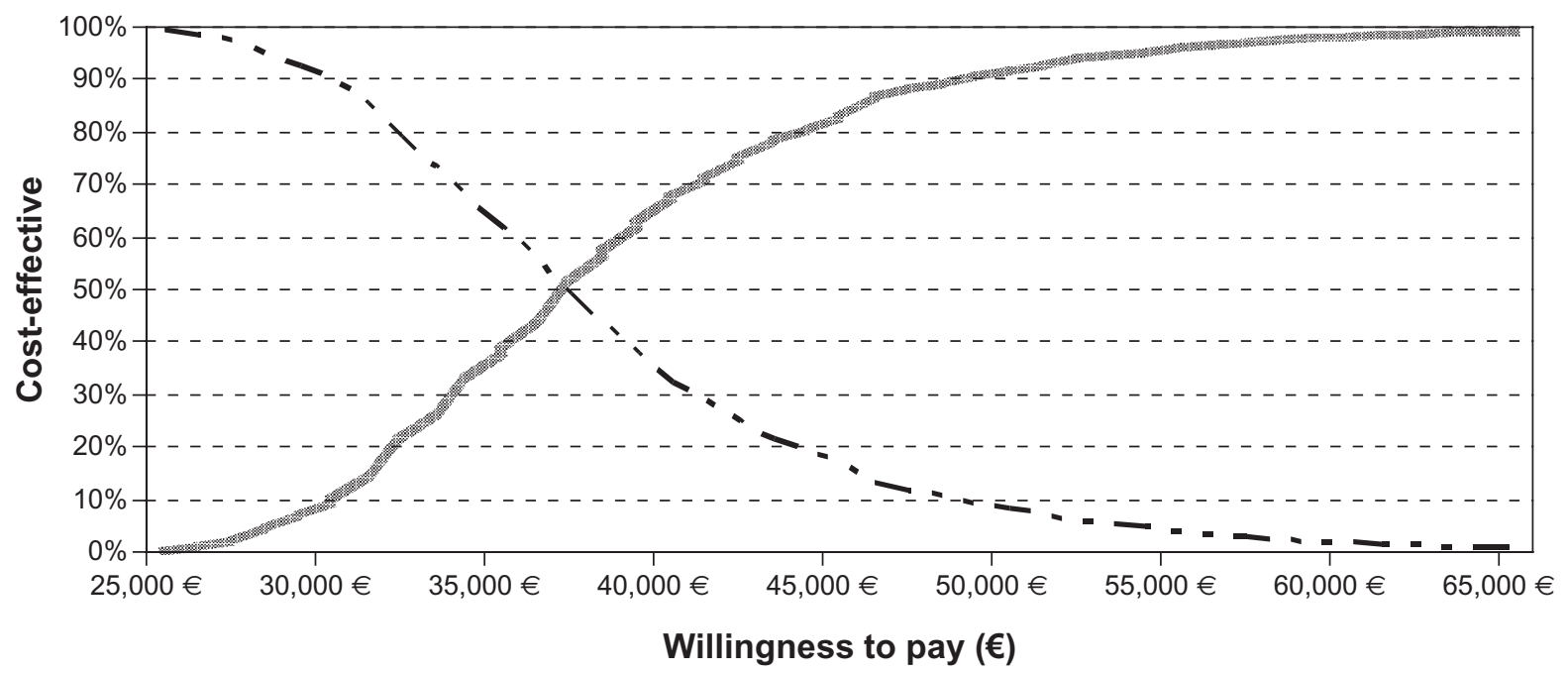

- - Bortezomib

Figure 3 Cost-effectiveness acceptability curve for lenalidomide-dexamethasone versus bortezomib. Abbreviation: Len/Dex, lenalidomide-dexamethasone.

gross domestic product per capita has been recommended as an acceptable threshold by the World Health Organization. ${ }^{32}$

According to provisional data, the gross domestic product per capita in the case of Greece was approximately $€ 20,000^{33}$ in 2011 and therefore $€ 60,000$ per QALY can be considered as an appropriate threshold in the context of the World Health Organization recommendation. Probabilistic analysis showed that, at this threshold, lenalidomide-dexamethasone may be cost-effective relative to bortezomib in $95 \%$ of cases. However, it must be noted that in the context of the current politicoeconomic crisis, Greece's coalition government agreed to a reform program with the International Monetary Fund, aiming to reduce the pharmaceutical and total public health care expenditure. ${ }^{34}$ In such a situation, and for an interim period, the unofficial willingness-to-pay threshold could be potentially lower than the threshold proposed by the World Health Organization.

Table 6 One-way sensitivity analysis for main model parameters

\begin{tabular}{|c|c|c|}
\hline & $\begin{array}{l}\text { Cost per } \\
\text { QALY (€) }\end{array}$ & $\begin{array}{l}\text { Cost per } \\
\text { life year }(€)\end{array}$ \\
\hline Base case & 38,268 & 29,415 \\
\hline All costs $+10 \%$ (drugs excluded) & 38,884 & 30,357 \\
\hline All costs - $10 \%$ (drugs excluded) & 36,984 & 28,271 \\
\hline Len-Dex cost $+10 \%$ & 48,026 & 36,344 \\
\hline Len-Dex cost $-10 \%$ & 29,910 & 22,989 \\
\hline Utilities $+10 \%(C R=0.9$ and $P D=0.7)$ & 38,018 & 29,251 \\
\hline Utilities $-10 \%(C R=0.7$ and $P D=0.57)$ & 38,686 & 29,609 \\
\hline
\end{tabular}

Abbreviations: CR, complete response; PD, progressive disease; Len-Dex, lenalidomide-dexamethasone; Bort, bortezomib.
Even in this situation, the combination of lenalidomidedexamethasone may still remain a cost-effective option from a pharmacoeconomic point of view at certain lower levels of willingness to pay.

It must be highlighted that pharmacoeconomic evaluations comparing different therapeutic options in this group of patients are limited in the literature, and the cost-effectiveness analysis of these agents needs further investigation. ${ }^{35}$ However, according to recent evidence, combination therapy with lenalidomide-dexamethasone may be a cost-effective option for the treatment of patients with rrMM in several European countries and settings. ${ }^{16,36,37}$ Concerning Greece, to the best of our knowledge, this is the first pharmacoeconomic study undertaken to compare these two agents.

It must be highlighted that the results of this model are not easily transferable ${ }^{38}$ and have to be considered strictly in the context of the Greek setting and on the basis of the present time and management practice with respect to patients, as well as drug and health resource prices. In addition, they have to be viewed in the context of the underlying model assumptions and data, which were derived from three trials and which may change in the future. There are common limitations in analysis using similar methodologies. They do not represent experimental research, but are instead based on simulation modeling and on data reported in the literature. ${ }^{39,40}$ To limit the possible sources of bias, standard recommendations were followed, namely conducting a systematic review, assessment of the evidence in collaboration with expert 
clinicians, and stochastic analysis in order to draw robust conclusions for the case of Greece.

As already mentioned, the analysis was based on the Phase III clinical trials MM-009 and MM-010, and APEX, and there are no direct trial comparisons between lenalidomidedexamethasone and bortezomib. Despite similarities in the target patient population, heterogeneity still exists between these studies. More specifically, in the studies comparing lenalidomide-dexamethasone to dexamethasone, the patients were older and were more heavily pretreated (about $7 \%$ of patients had previous bortezomib). Furthermore, APEX was an open-label study, while MM-009 and MM-010 were double-blind, placebo-controlled studies. Hence, despite the fact that these two groups have been used in previous economic studies, there is a concern about comparing a twodrug regimen with a single drug. For instance, it could be argued that a bortezomib-dexamethasone combination may yield different results in terms of time to progression and overall survival for a very small additional cost. However, this argument is questionable, given that the MMY-3002 study, which compared bortezomib with bortezomib-doxorubicin, produced similar results to APEX in terms of response rates and progression-free survival. Regarding the addition of dexamethasone, it is recognized that this improves response rates, but we lack Phase III data to support that this also translates into improved progression-free survival or overall survival. Even in a more recent Phase III study of subcutaneous versus intravenous administration of bortezomib in patients with relapsed multiple myeloma, bortezomib was used without dexamethasone, which was added only if there was no response to the initial therapy of bortezomib alone. ${ }^{41}$ Thus, for the purposes of this analysis, it would be difficult to extract data regarding the combination of bortezomib with dexamethasone in rrMM. Of course, a comparison with other bortezomib-based regimens in patients with rrMM could possibly yield different results, but in this case, three-drug or four-drug combination regimens are also associated with different toxicity and additional cost.

Another significant limitation is the lack of quality of life data associated with the treatment options in hand. In terms of administration, lenalidomide is given orally while bortezomib is given intravenously in hospital. Thus, patients on lenalidomide have a better quality of life; they also may require less staff and patient time, and in certain settings this may have an impact on providers, patient productivity, and direct medical costs. ${ }^{27}$ However, productivity losses and direct nonmedical patient costs were not considered here. Further, utility estimates for the response levels were based on the literature, so the QALYs were not those for the general Greek population.

The treatment of patients with rrMM is challenging, and both bortezomib and lenalidomide are major treatment options. However, in certain patients one may be preferable to the other due to factors such as previous neuropathy, in which case bortezomib may be relatively less desirable. ${ }^{42}$ On the other hand, in patients with renal dysfunction, especially of recent onset, bortezomib may be preferable. ${ }^{43}$ Regarding certain risk features, such as high-risk cytogenetics, bortezomib may be more effective in patients with del17p or $t(4 ; 14)$, but this is derived only from retrospective data and is subject to bias. ${ }^{43-45}$ Furthermore, the preferences of the patients should always be taken into account, especially considering the intravenous (or more recently subcutaneous) route of administration for bortezomib, need for hospital admission, and cost of traveling to a specialized center for bortezomib therapy.

\section{Conclusion}

A combination of lenalidomide and dexamethasone may be a cost-effective choice, so is good value for money, compared with bortezomib monotherapy, when used in previous treated multiple myeloma patients in the National Health Service of Greece, especially at willingness-to pay-thresholds above $€ 40,000$ per QALY gained.

\section{Acknowledgment}

The authors would like to thank Philip Lees, Technical Editor of the Hellenic Journal of Cardiology, for his critical assistance with the English text.

\section{Disclosure}

The present study was sponsored by Genesis Pharma Hellas. The authors declare no other conflicts of interest with this work.

\section{References}

1. Jemal A, Siegel R, Xu J, Ward E. Cancer statistics, 2010. CA Cancer J Clin. 2010;60:277-300.

2. Globocan. Country Fast Stats. 2008. Available from: http://globocan. iarc.fr/factsheets/populations/factsheet.asp?uno=968. Accessed September 12, 2012.

3. Becker N. Epidemiology of multiple myeloma. Recent Results Cancer Res. 2011;183:25-35.

4. Alegre A, Vicuna I, Aguado B. Safety and efficacy of lenalidomide in relapsed or refractory multiple myeloma. Clin Med Insights Oncol. 2012;6:1-10.

5. Ludwig H, Bolejack V, Crowley J, et al. Survival and years of life lost in different age cohorts of patients with multiple myeloma. J Clin Oncol. 2010;28:1599-1605.

6. Kumar SK, Rajkumar SV, Dispenzieri A, et al. Improved survival in multiple myeloma and the impact of novel therapies. Blood. 2008; 111:2516-2520. 
7. Brenner H, Gondos A, Pulte D. Recent major improvement in longterm survival of younger patients with multiple myeloma. Blood. 2008;111:2521-2526.

8. Brenner H, Gondos A, Pulte D. Expected long-term survival of patients diagnosed with multiple myeloma in 2006-2010. Haematologica. 2009; 94:270-275.

9. Kastritis E, Zervas K, Symeonidis A, et al. Improved survival of patients with multiple myeloma after the introduction of novel agents and the applicability of the International Staging System (ISS): an analysis of the Greek Myeloma Study Group (GMSG). Leukemia. 2009;23: 1152-1157.

10. Greenberg AJ, Vachon CM, Rajkumar SV. Disparities in the prevalence, pathogenesis and progression of monoclonal gammopathy of undetermined significance and multiple myeloma between blacks and whites. Leukemia. 2012;26:609-614.

11. Kvam AK, Fayers P, Hjermstad M, Gulbrandsen N, Wisloff F. Health-related quality of life assessment in randomised controlled trials in multiple myeloma: a critical review of methodology and impact on treatment recommendations. Eur J Haematol. 2009;83:279-289.

12. Gulbrandsen N, Hjermstad MJ, Wisloff F. Interpretation of quality of life scores in multiple myeloma by comparison with a reference population and assessment of the clinical importance of score differences. Eur $J$ Haematol. 2004;72:172-180.

13. Durie BG. New approaches to treatment for multiple myeloma: durable remission and quality of life as primary goals. Clin Lymphoma Myeloma. 2005;6:181-190

14. Engelhardt M, Kleber M, Udi J, et al. Consensus statement from European experts on the diagnosis, management, and treatment of multiple myeloma: from standard therapy to novel approaches. Leuk Lymphoma. 2010;51:1424-1443.

15. Cook R. Economic and clinical impact of multiple myeloma to managed care. J Manag Care Pharm. 2008;14(Suppl 7):19-25.

16. Moller J, Nicklasson L, Murthy A. Cost-effectiveness of novel relapsed-refractory multiple myeloma therapies in Norway: lenalidomide plus dexamethasone versus bortezomib. J Med Econ. 2011; 14:690-697.

17. Caro JJ. Pharmacoeconomic analyses using discrete event simulation. Pharmacoeconomics. 2005;23:323-332.

18. Weber DM, Chen C, Niesvizky R, et al. Lenalidomide plus dexamethasone for relapsed multiple myeloma in North America. N Engl J Med. 2007;357:2133-2142.

19. Dimopoulos M, Spencer A, Attal M, et al. Lenalidomide plus dexamethasone for relapsed or refractory multiple myeloma. $N$ Engl J Med. 2007;357:2123-2132.

20. Dimopoulos MA, Chen C, Spencer A, et al. Long-term follow-up on overall survival from the MM-009 and MM-010 phase III trials of lenalidomide plus dexamethasone in patients with relapsed or refractory multiple myeloma. Leukemia. 2009;23:2147-2152.

21. Richardson PG, Sonneveld P, Schuster MW, et al. Bortezomib or highdose dexamethasone for relapsed multiple myeloma. $N$ Engl J Med. 2005;352:2487-2498.

22. Richardson PG, Sonneveld P, Schuster M, et al. Extended follow-up of a phase 3 trial in relapsed multiple myeloma: final time-to-event results of the APEX trial. Blood. 2007;110:3557-3560.

23. Blade J, Samson D, Reece D, et al. Criteria for evaluating disease response and progression in patients with multiple myeloma treated by high-dose therapy and haemopoietic stem cell transplantation. Myeloma Subcommittee of the EBMT. European Group for Blood and Marrow Transplant. Br J Haematol. 1998;102:1115-1123.

24. Maniadakis N, Dafni U, Fragoulakis V, et al. Economic evaluation of taxane-based first-line chemotherapy in the treatment of patients with metastatic breast cancer in Greece: an analysis alongside a multicenter, randomized phase III clinical trial. Ann Oncol. 2009;20:278-285.

25. Maniadakis N, Fragoulakis V, Pallis A, Prezerakos P, Georgoulias V. Economic evaluation of docetaxel/gemcitabine versus docetaxel as frontline treatment of patients with advanced/metastatic non-small cell lung cancer in Greece. Lung Cancer. 2007;58:275-281.
26. Available from: http://www.yyka.gov. Accessed September 15, 2012.

27. Maniadakis N, Fragoulakis V, Pectasides D, Fountzilas G. XELOX versus FOLFOX6 as an adjuvant treatment in colorectal cancer: an economic analysis. Curr Med Res Opin. 2009;25:797-805.

28. van Agthoven M, Segeren CM, Buijt I, et al. A cost-utility analysis comparing intensive chemotherapy alone to intensive chemotherapy followed by myeloablative chemotherapy with autologous stem-cell rescue in newly diagnosed patients with stage II/III multiple myeloma; a prospective randomised phase III study. Eur J Cancer. 2004;40: 1159-1169.

29. Desgagne A, Castilloux AM, Angers JF, LeLorier J. The use of the bootstrap statistical method for the pharmacoeconomic cost analysis of skewed data. Pharmacoeconomics. 1998;13(5 Pt 1): 487-497.

30. McCabe C, Claxton K, Culyer AJ. The NICE cost-effectiveness threshold: what it is and what that means. Pharmacoeconomics. 2008; 26:733-744

31. Simoens S. Biosimilar medicines and cost-effectiveness. Clinicoecon Outcomes Res. 2011;3:29-36.

32. Zhao FL, Yue M, Yang H, Wang T, Wu JH, Li SC. Willingness to pay per quality-adjusted life year: is one threshold enough for decisionmaking?: results from a study in patients with chronic prostatitis. Med Care. 2011;49:267-272.

33. Hellenic Statistical Authority. Available from: http://www.statistics.gr. Accessed September 15, 2012.

34. Fragoulakis V, Kourlaba G, Goumenos D, Konstantoulakis M, Maniadakis N. Economic evaluation of intravenous iron treatments in the management of anemia patients in Greece. Clinicoecon Outcomes Res. 2012;4:127-134.

35. Messori A, Maratea D, Nozzoli C, Bosi A. The role of bortezomib, thalidomide and lenalidomide in the management of multiple myeloma: an overview of clinical and economic information. Pharmacoeconomics. 2011;29:269-285.

36. Brown RE, Stern S, Dhanasiri S, Schey S. Lenalidomide for multiple myeloma: cost-effectiveness in patients with one prior therapy in England and Wales. Eur J Health Econ. May 10, 2012. [Epub ahead of print.]

37. Félix J, Almeida J, Vandewalle B. A comprehensive costeffectiveness analysis of lenalidomide for multiple myeloma patients who have received at least one prior therapy. Value Health. 2012; 15:A218

38. Drummond M, Barbieri M, Cook J, et al. Transferability of economic evaluations across jurisdictions: ISPOR Good Research Practices Task Force report. Value Health. 2009;12:409-418.

39. Picot J, Cooper K, Bryant J, Clegg AJ. The clinical effectiveness and cost-effectiveness of bortezomib and thalidomide in combination regimens with an alkylating agent and a corticosteroid for the first-line treatment of multiple myeloma: a systematic review and economic evaluation. Health Technol Assess. 2011;15:1-204.

40. Gaultney JG, Redekop WK, Sonneveld P, Uyl-de Groot CA. Novel anticancer agents for multiple myeloma: a review of the evidence for their therapeutic and economic value. Expert Rev Anticancer Ther. 2012;12:839-854

41. Moreau P, Pylypenko H, Grosicki S, et al. Subcutaneous versus intravenous administration of bortezomib in patients with relapsed multiple myeloma: a randomised, phase 3, non-inferiority study. Lancet Oncol. 2011;12:431-440.

42. Dimopoulos MA, Kastritis E, Christoulas D, et al. Treatment of patients with relapsed/refractory multiple myeloma with lenalidomide and dexamethasone with or without bortezomib: prospective evaluation of the impact of cytogenetic abnormalities and of previous therapies. Leukemia. 2010;24:1769-1778.

43. Dimopoulos MA, Roussou M, Gkotzamanidou M, et al. The role of novel agents on the reversibility of renal impairment in newly diagnosed symptomatic patients with multiple myeloma. Leukemia. 2013;27: 423-429. 
44. Kapoor P, Fonseca R, Rajkumar SV, et al. Evidence for cytogenetic and fluorescence in situ hybridization risk stratification of newly diagnosed multiple myeloma in the era of novel therapie. Mayo Clin Proc. 2010;85:532-537.
45. Reece D, Song KW, Fu T, et al. Influence of cytogenetics in patients with relapsed or refractory multiple myeloma treated with lenalidomide plus dexamethasone: adverse effect of deletion 17p13. Blood. 2009;114: $522-525$.

\section{Publish your work in this journal}

Cancer Management and Research is an international, peer-reviewed open access journal focusing on cancer research and the optimal use of preventative and integrated treatment interventions to achieve improved outcomes, enhanced survival and quality of life for the cancer patient. The journal welcomes original research, clinical \& epidemiological studies, reviews \& evaluations, guidelines, expert opinion \& commentary, case reports \& extended reports. The manuscript management system is completely online and includes a very quick and fair peerreview system, which is all easy to use. Visit http://www.dovepress.com/ testimonials.php to read real quotes from published authors. 